\section{Isotopic Composition of Boron and its Atomic Weight}

AFTER an extensive investigation, we conclude that a boron- 11 /boron- 10 ratio of 4.00 (that is, $80 / 20$ ) approaches the true natural abundance ratio much more closely than the presently 'accepted' value of $4 \cdot 31(81 \cdot 17 / 18 \cdot 83)$. The latter figure is based on a mass spectrometric investigation of boron trifluoride ${ }^{1}$, a notoriously troublesome compound, in view of its adsorption characteristics resulting in instrumental 'memory'. Since publication of that work a number of attempts to verify the abundance ratio from boron trifluoride have resulted in a wide range of values.

On the other hand, abundance ratios calculated by various investigators from mass spectra of boron hydrides have been highly consistent, yielding ratios of approximately $4 \cdot 00$. Lest it be argued that this lower value may be peculiar to boron hydrides, we would like to point out that recent work in this laboratory with other compounds (for example, trimethylboroxine) has yielded the same ratio.

We therefore propose that in the future it would be far more reasonable to use the ratio $4.00(80 / 20)$ to represent the natural isotopic abundance of boron. This would require in addition a revision of the atomic weight from $10 \cdot 82$ to $10 \cdot 811$, a change which seems well within the range of experimental error of earlier investigators ${ }^{2}$.

\section{W. J. LehManN}

I. Shapiro*

Research Laboratory,

Olin Mathieson Chemical Corporation,

Pasadena, California. March 11.

* Present address: I. Shapiro, Consultant, Box 24231, Los Angeles 24, California.

${ }^{1}$ Inghram, M., Phys. Rev., 70, 653 (1946).

${ }^{2}$ German Atomic Weight Commission, Ber. deutsch. chem. Ges., 56A, Uhemical Elements, J. Amer. Chem. Soc., 47, 597 (1925).

\section{BIOCHEMISTRY}

\section{Synthesis of Oxytocin by the Nitrophenyl Ester Method}

After the completion of work on the degration of oxytocin ${ }^{1}$, this compound was synthesized in this laboratory ${ }^{2}$. Since that time several other synthetic methods leading to biologically active solutions of the crude hormone have been reported ${ }^{3}$. Recently, an improved synthesis of oxytocin was elaborated ${ }^{4}$ by us. All these syntheses of oxytocin have the common feature of linking peptides together. It is well known, however, that formation of the peptide bond with a protected peptide as the acid component can lead to racemization ${ }^{5}$, the extent of which depends on the components, the conditions and the method. No racemization was observed with the use of the azide method ${ }^{6}$. Nevertheless, the latter procedure can involve the formation of by-products other than diastereoisomers. To avoid racemization it is possible to take advantage of the protecting groups, for example, the carbobenzoxy group, which protects the amino-acid acylated by it not only against unwanted acylation but also against racemization. This protection is effective only if the carbobenzoxy group is joined to the amino-acid, the carboxyl group of which takes part in the peptide bond-forming reaction. Therefore it becomes necessary to start the synthesis with the C-terminal amino-acid, to link to it the next (protected) aminoacid, to remove the protecting group from the resulting dipeptide derivative, to couple to the free amino component thus formed the third (protected) aminoacid, etc. This manner of peptide-chain synthesis is practicable only if the method used for the formation of the peptide bond is unambiguous, leads to only one peptide, and gives high yields. All the mixed anhydride methods were eliminated as they usually

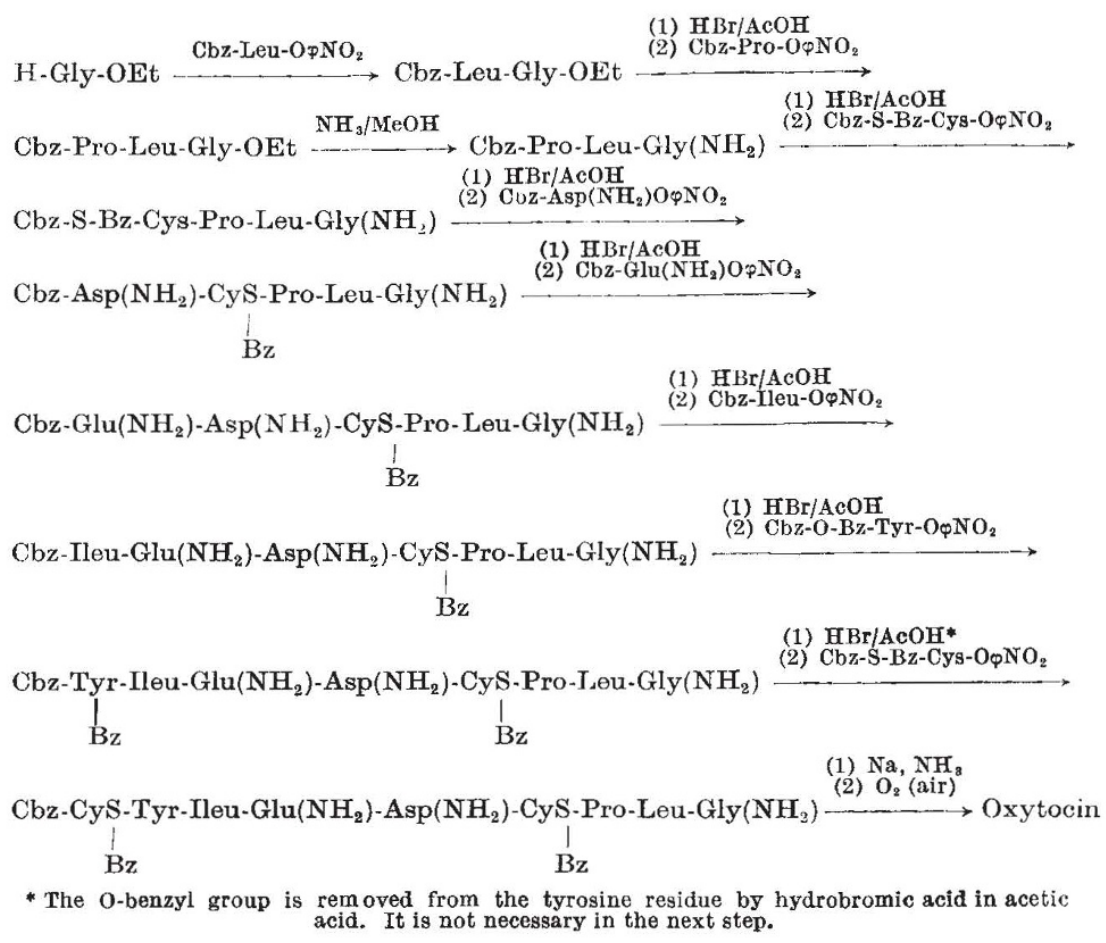

Fig. 1 